\title{
Tecnología CAD CAM aplicada al diseño de robots de batalla
}

CAD CAM technology applied to the design of battle robots.

Miguel Ángel Escobar Guachambala. ${ }^{1}$, Sócrates Miguel Aquino Arroba. ${ }^{2}$, Edwin Rodolfo Pozo Safla. ${ }^{3} \&$ Luis Santiago Choto Chariguaman. ${ }^{4}$

\begin{abstract}
.
DOI: https://doi.org/10.33262/cienciadigital.v3i3.2.721

A methodology is proposed for the computer-aided design and manufacturing of battle robot 12-pound, taking into account parameters and guidelines that regulate this category of robots, the objective is to determine the design of a battle robot with a low weight, functionality and to easy of construction, in order to guaranteed operability in the competition. Frame is the biggest robot'size and it was built in nylon, with the particularity that it was covered externally with aeronautical aluminum sheet, this method reduce the weight and it is guaranteed that the frame could absorb the hits emitted by the adversary The robot's weapon is type cam with an impact point, it was built in stainless steel martensitic.

For the construction of robot it was necessary to generate the detailed drawings, in addition to handling 3D CAD files for computer-aided manufacturing (CAM); the manufacturing processes used were: $\mathrm{CNC}$ machining, 3D printing, plasma cutting, heat treatment.
\end{abstract}

1 Escuela Superior Politécnica de Chimborazo, Facultad de Mecánica. Riobamba, Ecuador. maescobar@espoch.edu.ec

2 Escuela Superior Politécnica de Chimborazo, Facultad de Mecánica. Riobamba, Ecuador. saquino@espoch.edu.ec

3 Escuela Superior Politécnica de Chimborazo, Facultad de Mecánica. Riobamba, Ecuador. edwin.pozo@espoch.edu.ec

4 Escuela Superior Politécnica de Chimborazo, Facultad de Mecánica. Riobamba, Ecuador. lchoto@espoch.edu.ec 
Finally, a functional battle robot with a total weight of 11.8 pounds was obtained depending on the regulations for participation in competition events.

Keywords: Technology, battle robot, CAD, CAM.

\section{Resumen.}

Se plantea una metodología para el diseño y manufactura asistida por computador de un robot de batalla de 12 libras, teniendo en cuenta parámetros y lineamientos que regulan esta categoría de robots, el objetivo es determinar el diseño de un robot de batalla con un bajo peso, funcional y de fácil construcción, de tal manera que se garantice operatividad del mismo en la competición. El elemento de mayor tamaño del robot es el bastidor y se construyó en nylon, con la particularidad que se recubrió externamente con chapa de aluminio aeronáutico, de esta manera de redujo el peso y se garantiza que el bastidor pueda absorber los golpes emitidos por el adversario. El arma del robot es tipo leva con un punto de impacto, fue construida en acero inoxidable martensitico.

Para la construcción del robot fue necesario generar los planos de detalle, además de manejar archivos CAD 3D para la manufactura asistida por computador (CAM); los procesos de manufactura utilizados fueron los siguientes: mecanizado CNC, impresión en 3D, corte por plasma, tratamiento térmico.

Finalmente se obtuvo un robot de batalla funcional con un peso total de 11.8 libras en función de la reglamentación para participación en eventos de competición.

Palabras Clave: Tecnología, robot de batalla, CAD, CAM.

\section{Introducción.}

El desarrollo de un producto mediante software CAD CAM es muy utilizado, porque facilita el diseño, construcción, ensamble, estudio de movimiento y manufactura asistida por computador. [1] En la actualidad se pueden realizar prototipos rápidos mediante el empleo de la manufactura aditiva, esto permite obtener una apreciación de la geometría y funcionalidad del elemento de máquina. Con el empleo de estas tecnologías se pueden obtener productos de bajo costo, calidad y competitivos en el mercado.[2]

Es un complejo sistema o máquina, que tiene como finalidad, luchar en un ring contra otro semejante, utilizando todo tipo de armas y equipamientos que las reglas del torneo en específico lo permitan. Los combates de robots se basan en la competición donde robots diseñados, construidos o programados por diferentes participantes compiten según un conjunto determinado de reglas.[3] 
El diseño y construcción de robot de batalla permite a los estudiantes y profesionales de ingeniería aplicar los conocimientos de $\mathrm{CAD}$, materiales, mecanismos, diseño de máquinas, electrónica, CAD CAM CAE y demás de otros conocimientos; cada diseñador presenta diseños innovadores y vanguardistas de robots, dotando de diferentes sistemas de transmisión de movimiento, defensa y de ataque.[4] El presente robot de batalla está diseñado en función del estudio del arte de diferentes robots que han sido probados en eventos de competencia y que han tenido éxito en las categorías que han participado.[5] A partir de esta información obtenida, se ha combinado las ventajas de cada uno de los robots estudiados, con lo cual se ha llegado a un diseño eficiente y en función de la reglamentación de eventos de competencia de robots de batalla. En la figura 1, se observa el diseño de robot que ha demostrado un buen desempeño en combates de las categorías de 1, 3, 12 y 120 libras.

Figura. 1. Robot batalla-Armagedrum 12 libras

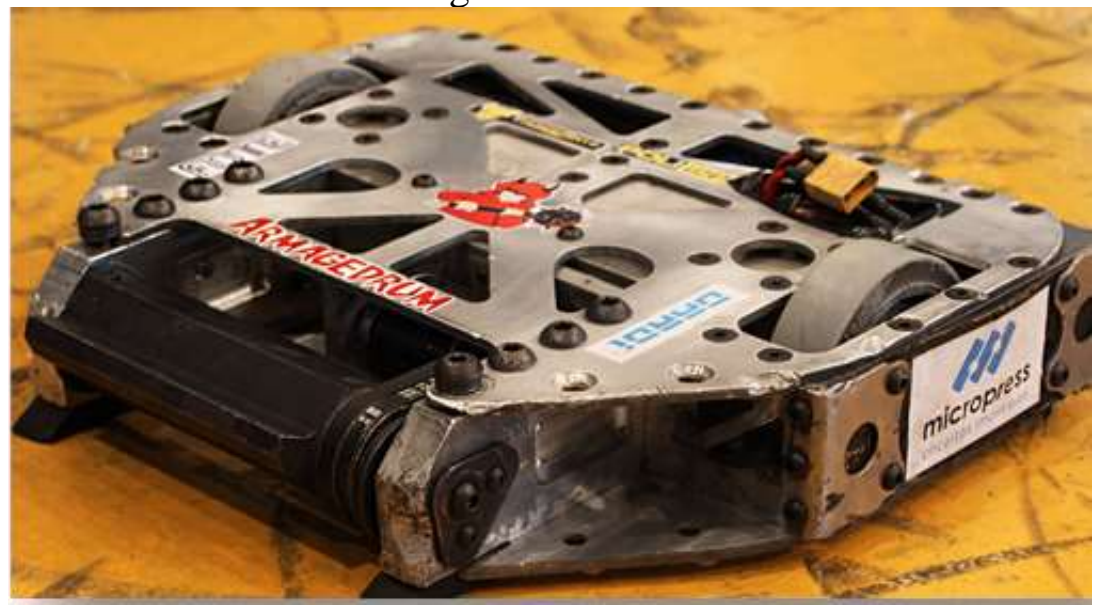

Fuente: THUNDERATZ, Brasil.

\section{Metodología}

Las etapas que se desarrollan para llegar al diseño final del robot de batalla se muestran en la figura 2.

Figura. 2 Diseño y construcción de un robot de batalla.

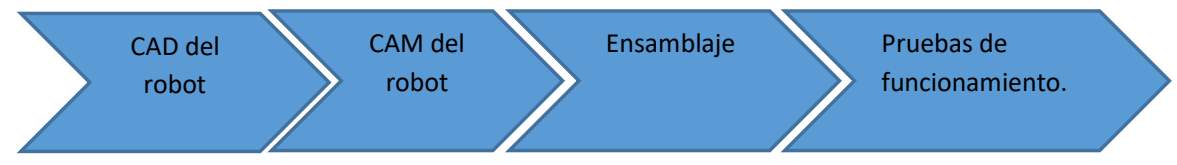

\section{Resultados}

\section{Diseño asistido por computador del robot de batalla 12 libras.}

El robot de batalla está diseñado en función del estudio de diferentes robots reconocidos por su gran desempeño en eventos de competencia, a partir de la información recabada, se ha 
combinado las ventajas de cada uno de los robots estudiados, con lo cual se ha llegado a un diseño en función de la reglamentación de eventos de competencia de robots de batalla.[6]

Mediante la utilización de software CAD se desarrolló un robot de batalla para participar en la categoría de 12 libras, lo más importante a considerar fue el peso del robot, con el fin de calificar en la categoría deseada.

En esta propuesta de robot se implementó un sistema de transmisión de movimiento rápido para tener mayor agilidad y maniobrabilidad en la arena, mediante la implementación de servomotores de alta velocidad y torque, además el arma fue diseñada con una geometría similar a una leva con un punto de impacto y con un material de alta resistencia. [7] En la figura 3 , se observa el diseño final del robot de batalla.

Figura. 3 Robot batalla EIM, 12libras

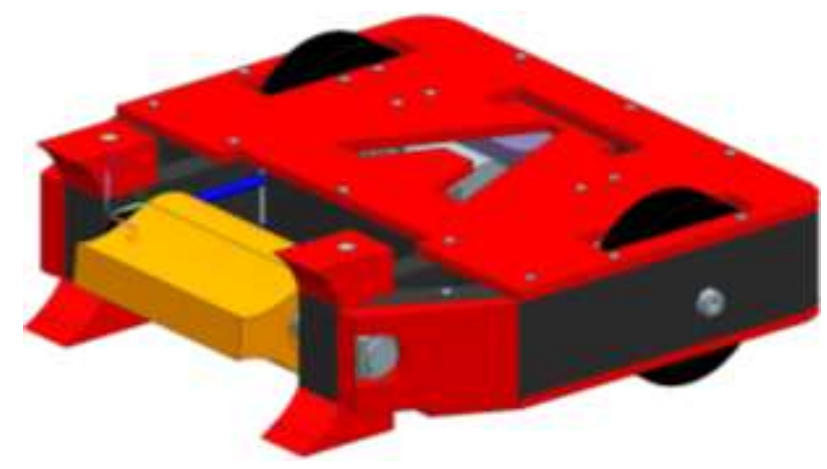

El software CAD nos ayudó eficientemente en la optimización de los espacios disponibles para el montaje de los motores, bridas, baterías, sistema electrónico y cableado del robot, con el objetivo reducir el peso.[8] En la figura 4, se observa la electrónica y demás accesorios del robot.

Figura. 4 Accesorio y sistema electrónico, robot batalla EIM, 12libras.

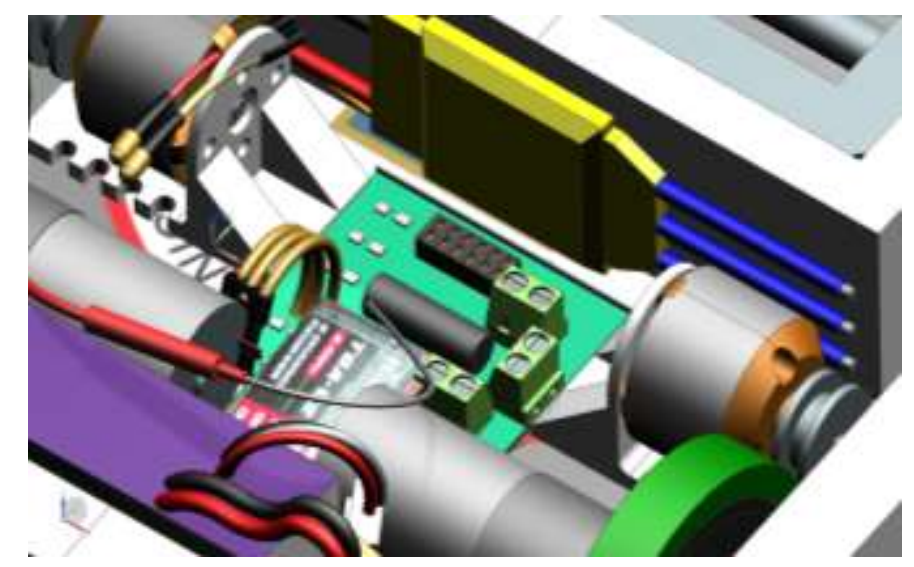




\section{Manufactura asistida por computador del robot batalla.}

En la figura 5, se observa las trayectorias de mecanizado del proceso de acabado del bastidor mediante software CAM, mediante esta tecnología se puede fabricar los elementos del robot sin ningún contratiempo, con alta precisión y calidad.

Figura. 5 Trayectorias de mecanizado CNC para el bastidor.

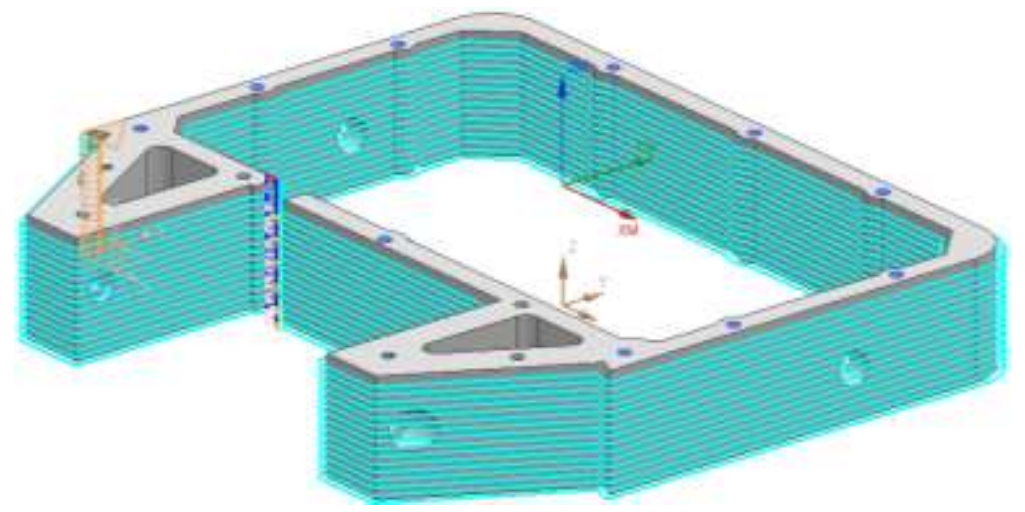

Una de las partes principales mecanizadas mediante $\mathrm{CNC}$ fue el bastidor, que está diseñado para contener todas las partes del robot, el material mecanizado fue el nylon, por tener buenas propiedades de maquinabilidad. [9] Las estrategias de mecanizado empleadas en la construcción del elemento mecánico fueron las siguientes: taladrado para las perforaciones roscadas, fresado de cavidades en el desbaste, contorneado para dar la forma final y un proceso de machuelado para tornillo M3. Todas las operaciones anteriormente mencionadas se las realizaron en un centro de mecanizado CNC 3 ejes, como se observa en la figura 6.

Figura. 6 Mecanizado $\mathrm{CNC}$ ejes del bastidor.

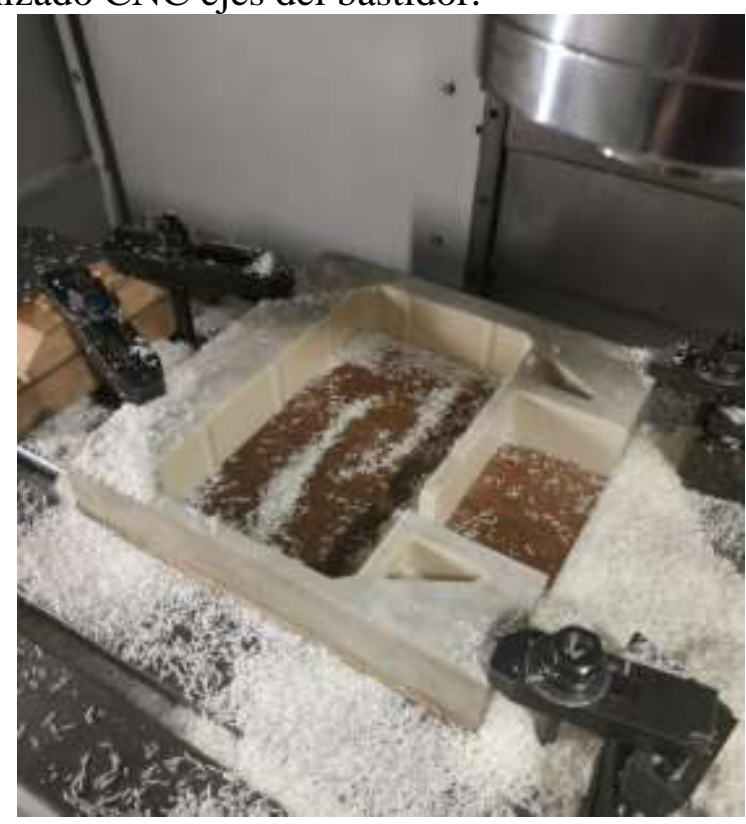


En la figura 7, se puede apreciar el mecanizado del arma rotatoria del robot, este elemento mecánico fue construido con acero inoxidable martensitico. Para mecanizar la geometría de arma, se empleó los siguientes procesos de mecanizado: taladrado para el alojamiento del eje, mecanizado de desbaste y afinado por contorno, además de cajeras para los alojamientos con ajuste de los rodamientos. [10]

Figura. 7 Mecanizado del arma del robot batalla.

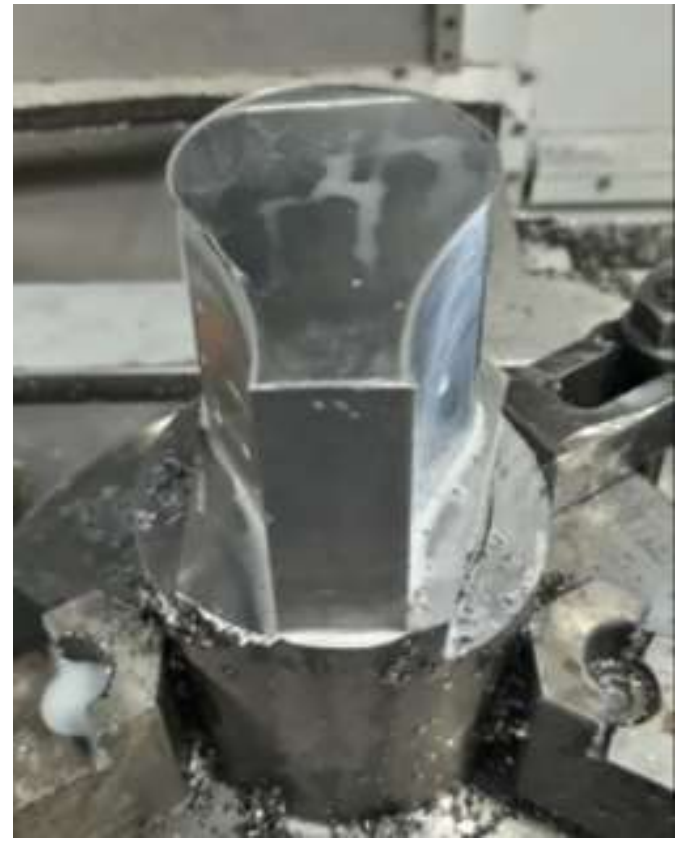

\section{Discusión}

A continuación se detallan los principales equipos y materiales que se emplearon en la construcción y ensamblaje del robot de batalla 12 lb, según tabla 1 .

Tabla 1. Componentes del robot de batalla.

\begin{tabular}{lc}
\hline COMPONENTES & Cantidad \\
\hline Sabertooth dual 5A motor driver & 1 \\
Driver motor Brushless & 2 \\
Moto reductor 26:1 & 2 \\
Motor Brushless 2,5 hp Escorpión & 2 \\
Batería LiPo 5s 75c & 1 \\
CR inalámbrico T6A-V2 de 2.4GHz & 1 \\
Bloque de nylon 300x300x50 & 1 \\
Chapa aluminio naval & 1 \\
Poleas de aluminio & 1 \\
Rodamientos de bola, Ø ext.19mm & 2 \\
Barra de acero inox. Ø2pulg. long. 80mm & 1 \\
Llantas 2" 7/8 & 1 \\
\hline
\end{tabular}


La parte principal del robot es el bastidor, que cumple con la función de acoger a todos los elementos fijos y móviles del robot batalla; además cumple con la función de absorber la mayor cantidad de golpes generados por el contrincante durante la competencia, por lo cual el bastidor fue cubierto con chapa de aluminio aeronáutico. En la figura 8, se observa la geometría del bastidor.

Figura. 8 Bastidor del robot batalla

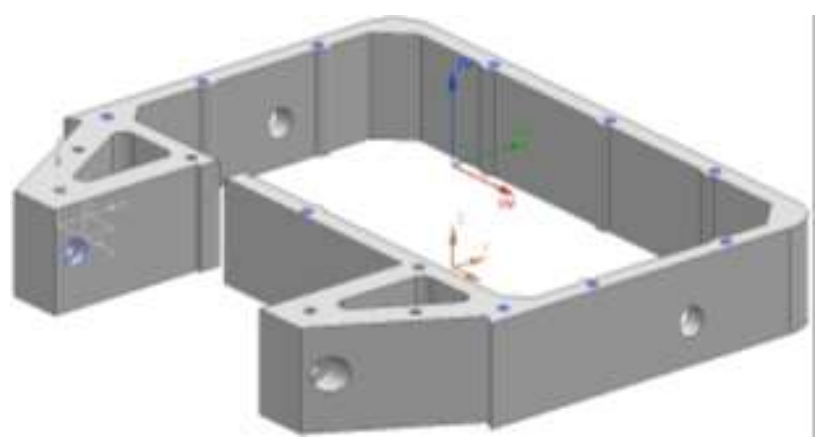

$\begin{array}{ll}\text { Denominación } & \text { BASTIDOR } \\ \text { Dimensiones } & 285 \times 249 \times 46 \mathrm{~mm} \\ \text { Material } & \text { Nylon } \\ \text { Peso } & 1.691 \mathrm{~b} \\ \text { Cantidad } & 1 \text { unidad }\end{array}$

En la figura 9, se observa la geometría de arma del robot, es similar a una leva con un punto de impacto, este elemento mecánico gira alrededor de dos rodamientos de carga radial de bolas; la transmisión del movimiento desde el motor hacia el arma se genera mediante un sistema de polea y banda.

Figura. 9 Arma rotatoria con un punto de impacto.

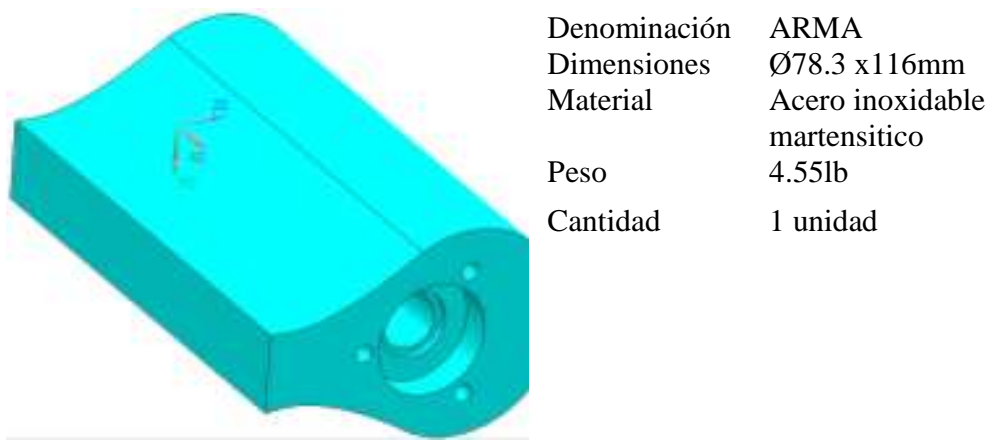

En la figura 10, se observa la tapa mecanizada en nylon, tiene la función de proteger el sistema electrónico, motores, baterías y otros accesorios de importancia para el funcionamiento del robot. La construcción se realizó mediantes placas de nylon, posteriormente se cubrió con chapa de aluminio aeronáutico. 
Figura. 10 Tapa del bastidor.

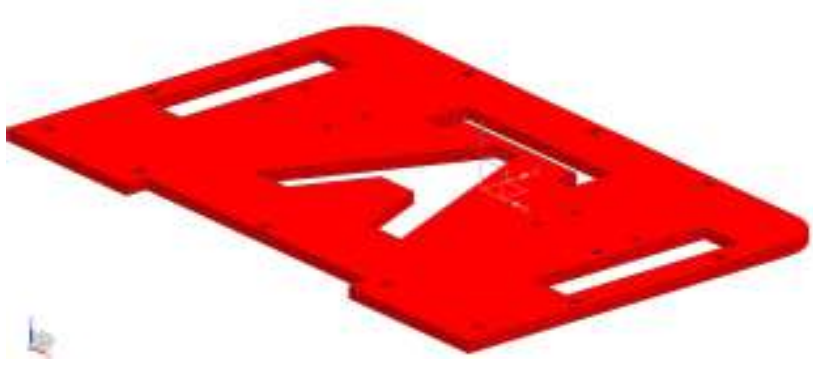

$\begin{array}{ll}\text { Denominación } & \text { TAPA } \\ \text { Dimensiones } & 281 \times 151 \times 5 \mathrm{~mm} \\ \text { Material } & \text { Nylon } \\ \text { Peso } & 0.55 \mathrm{lb} \\ \text { Cantidad } & 2 \text { unidades }\end{array}$

En la figura 11, se observa una polea de duraluminio, especialmente diseñada para acoplarse a la arma mediante perno de sujeción M3, de tal forma que pueda transmitir el movimiento desde el motor hasta el arma mediante banda.

Figura. 11 Polea

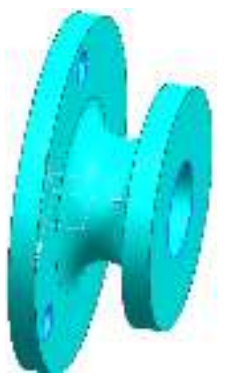

$\begin{array}{ll}\text { Denominación } & \text { Polea } \\ \text { Dimensiones } & \varnothing 44 \times 16 \mathrm{~mm} \\ \text { Material } & \text { Duraluminio } \\ \text { Peso } & 0.045 \mathrm{lb} \\ \text { Cantidad } & 2 \text { unidades }\end{array}$

En la figura 12, se observa una rueda mecanizada en caucho sintético, mediante un torno CNC, para evitar la necesidad de importar ruedas normalizadas.

Figura. 12 Rueda

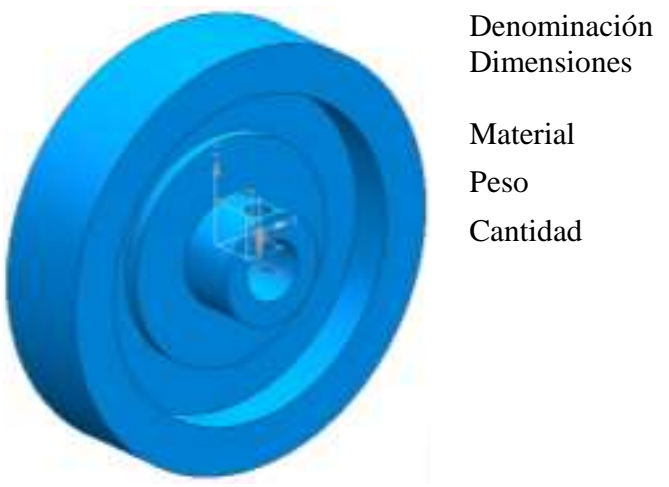

Rueda

Ø2-7/8 pulg.

Espesor $27 \mathrm{~mm}$

Caucho sintético.

$0.16 \mathrm{lb}$

2 unidades 
$\mathrm{Al}$ finalizar el proceso de mecanizado $\mathrm{CNC}$ de las diferentes partes diseñadas para el robot, se procedió al ensamblaje del robot y sus respectivas pruebas de funcionamiento. El robot de batalla que se construyo tiene un peso total de 11.8 libras, y en conclusión está homologado para participar en la categoría de 12 libras.

\section{Conclusiones.}

- El sistema de transmisión de movimiento debe estar dotado de ruedas resistentes, que estén posicionadas al interior del bastidor y además que parte de la rueda pueda superar el nivel superior e inferior del bastidor, con el objetivo principal de tener movilidad aún después de haber sido volteado por el contrincante.

- El peso del robot debe estar concentrado en un $75 \%$ sobre las ruedas posteriores, para obtener mayor agarre de las ruedas en la pista del combate, además se debe implementar un sistema de movilidad para la parte delantera del robot mediante esferas de rodadura, con el objetivo de reducir la fricción, permitiendo mayor maniobrabilidad y evasión de golpes.

- El bastidor debe ser construido de un material ligero, resistente, y diseñado en un cuerpo único para una mejor absorción de impactos. Para dar mayor resistencia a impactos el bastidor debe ser cubierto con una chapa ligera de alta resistencia al corte e impacto.

- Las armas giratorias con un lado de impacto, deben tener su centro de gravedad bien establecido, para evitar vibraciones en el robot a altas revoluciones.

- Las variantes de diseño y armas que se puede implementar en un robot de batalla dependen de la categoría en la cual va a participar, las categorías con mayor demanda son las de 1, 3, 12 y 120 libras. A mayor peso, se tiene facilidad para realizar diseños de robots que puedan contener mecanismos especiales de defensa y ataque.

\section{Referencias bibliográficas.}

[1] Richard A Gizelbach, CNC Machining. 2018.

[2] D. T. Diaz, "Tecnologías de Fabricación Digital Aditiva, ventajas para la construcción de modelos, prototipos y series cortas en el proceso de diseño de productos," Iconofacto, vol. 12, no. 18, pp. 118-143, 2016.

[3] R. C. Arkin, "Ethical robots in warfare," IEEE Technol. Soc. Mag., vol. 28, no. 1, pp. 30-33, 2009.

[4] P. Singer, "Robots at war," Wilson Q., 2008.

[5] P. Asaro, "How just could a robot war be," Curr. issues Comput. Philos., vol. 175, pp. 50-64, 2008.

[6] J. J. Shah and M. Mäntylä, Parametric and feature-based CAD/CAM: concepts, techniques, and applications. John Wiley \& Sons, 1995.

[7] F. J. Díaz, B. Tzancoff, M. Claudia, E. S. Martin, and F. López, "Aprendiendo a 
programar con juegos y robots," in VII Congreso de Tecnología en Educación y Educación en Tecnología, 2012.

[8] R. L. Norton, Diseño de máquinas. Pearson:, 1999.

[9] S. Kalpakjian and S. Schmid, "Manufacturing Processes," Pearson, 2008.

[10] T. Garcés, A. Xavier, and E. R. Lozano Granda, "Diseño y Construcción del un Robot de Batalla Controlado Mediante Dispositivo Bluetooth.," 2010. 


\section{PARA CITAR EL ARTÍCULO INDEXADO.}

Escobar Guachambala, M., Aquino Arroba, S., Pozo Safla, E. R., \& Choto Chariguaman, L. (2019).

Tecnología CAD CAM aplicada al diseño de robots de batalla. Ciencia Digital, 3(3.2), 139-149.

https://doi.org/10.33262/cienciadigital.v3i3.2.721

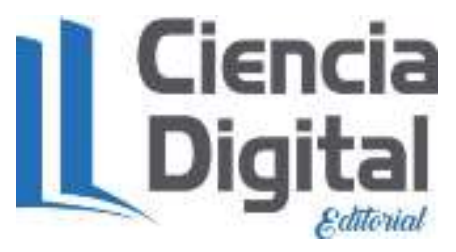

El artículo que se publica es de exclusiva responsabilidad de los autores y no necesariamente reflejan el pensamiento de la Revista Ciencia Digital.

El artículo queda en propiedad de la revista y, por tanto, su publicación parcial y/o total en otro medio tiene que ser autorizado por el director de la Revista Ciencia Digital.
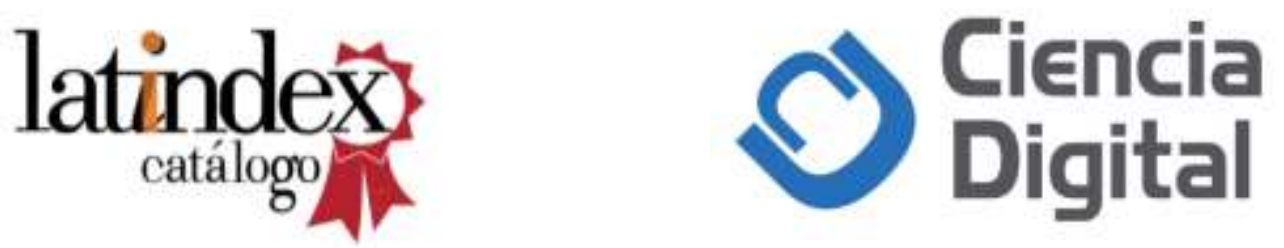